\title{
CURVED SENSORS
}

Performance of CMOS prototypes and wide field related imagers
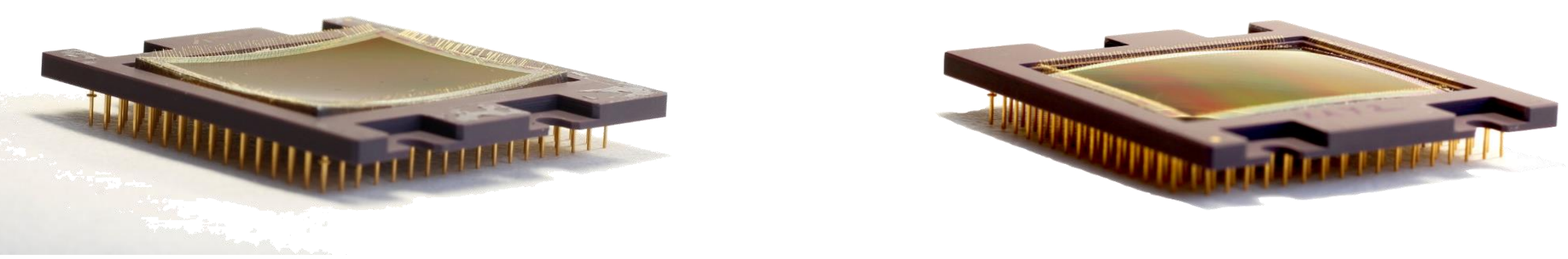

CNRS-INSU - Laboratoire d'Astrophysique de Marseille

Emmanuel HUGOT - Marc FERRARI - Simona LOMBARDO - Wilfried JAHN*

Mélanie ROULET - Thibault BEHAGHEL - Kelly JOAQUINA

CEA-LETI: Packaging \& Assembling Lab.

Bertrand CHAMBION - Christophe GASCHET - David HENRY 


\section{Curved sensors : why?}

\section{(Aix Marseille

Wide field images using curved photographic plates

Large Magellanic Cloud

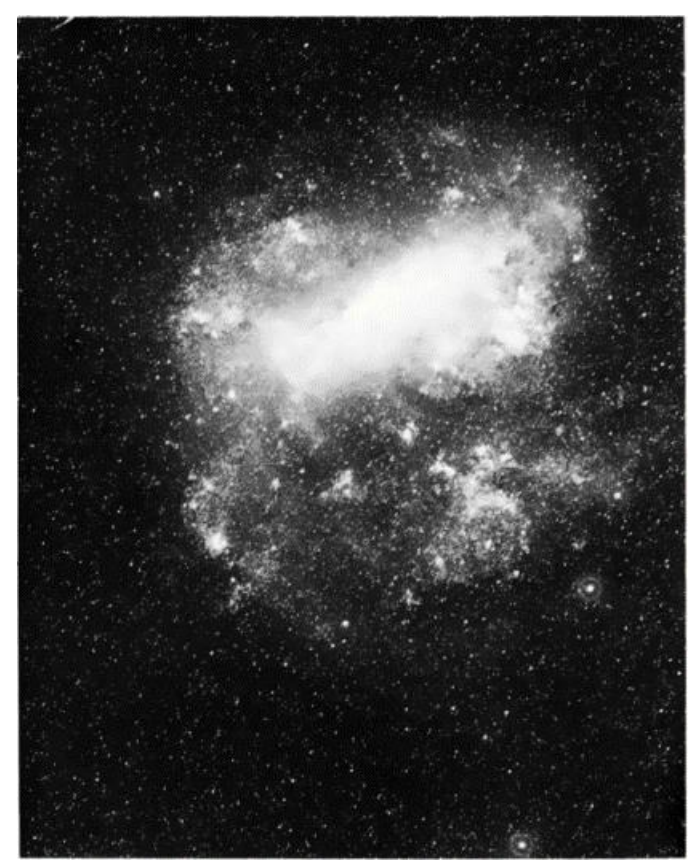

Credit: The Unity of Universe Book
Invention of CDD (1969) Numerical flat sensors

Complexification of the optics to flatten the image

Complexification of the focal plane

With curved sensors Back to simple optical systems !
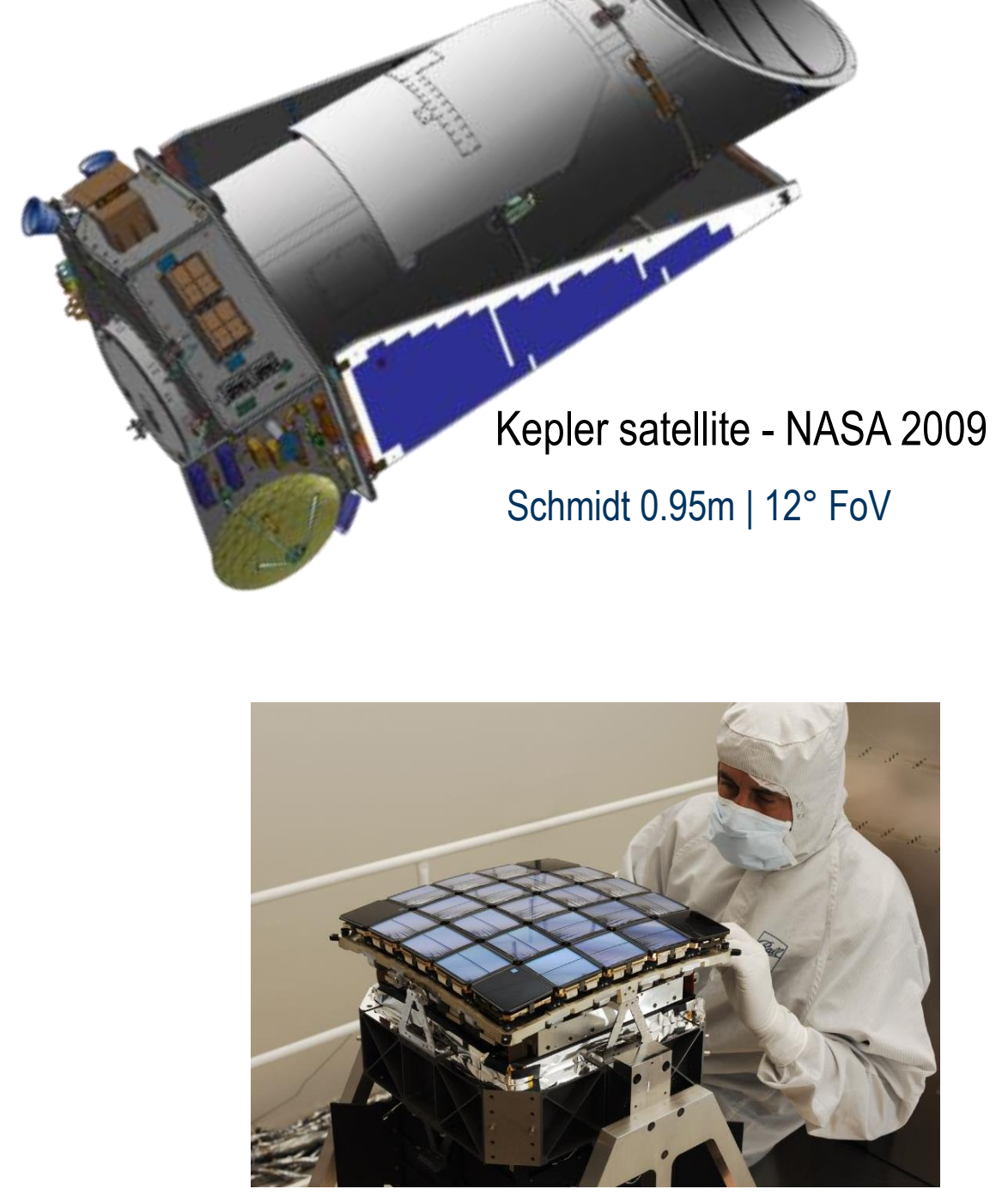

42 flat CDD 2k x 4k , 95 Mpx, on curved focal plane structure $\mathrm{R} \sim 1400 \mathrm{~mm}$ 


\section{(Aix Marseille

\section{Curved sensors : why?}

No field curvature correction $\rightarrow$ Less Optics

Gain in term of mass and size !

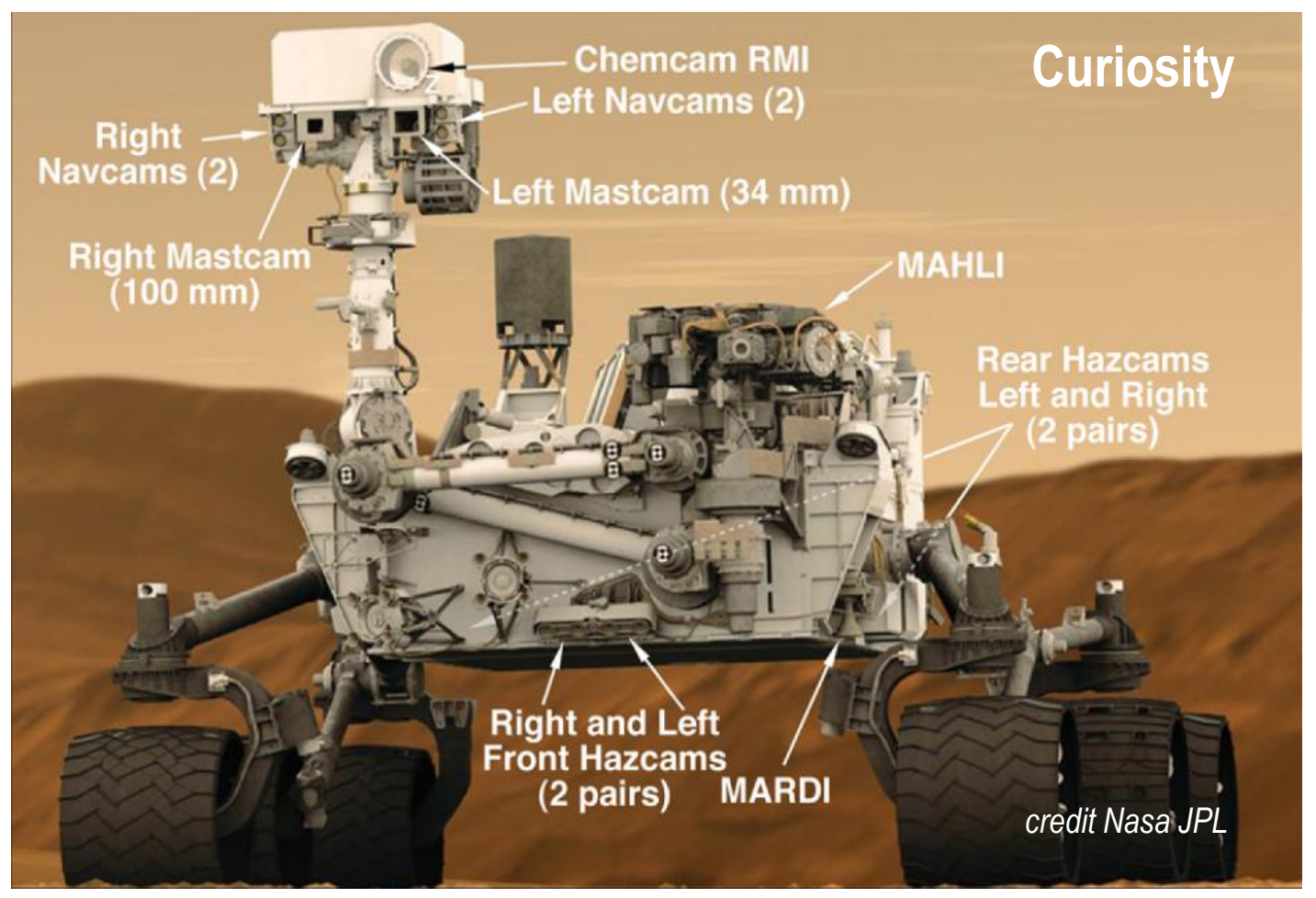

Very interesting for small payloads where every $(K) g$ counts !

Wide-angle $\left(180^{\circ}\right)$ : Fisheye cameras

Vision-angle $\left(40-70^{\circ}\right)$ : compact cameras

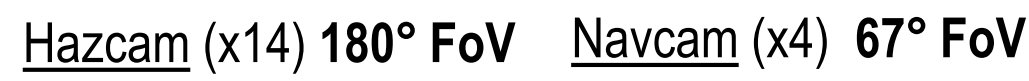
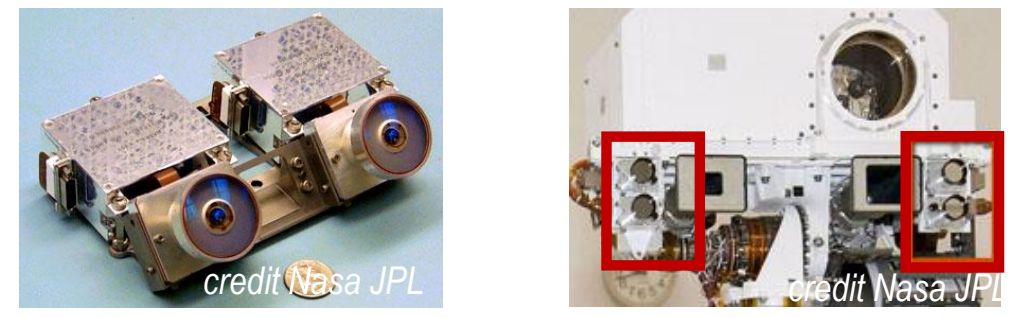

Mahli (x2) $40^{\circ} \mathrm{FoV}$

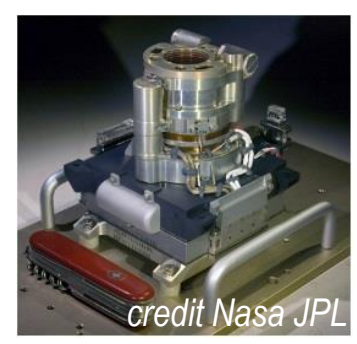

Mastcam (x2) $8.5^{\circ}$ and $25^{\circ}$

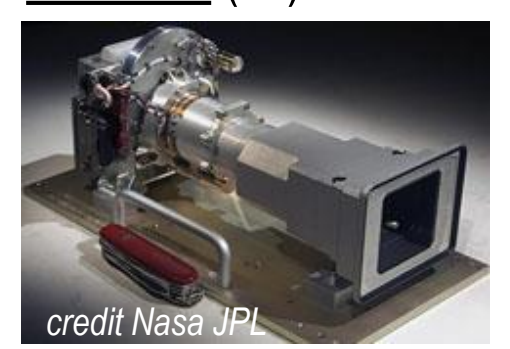


Full frame $24 \times 32 \mathrm{~mm}^{2}$ 20 Megapixels

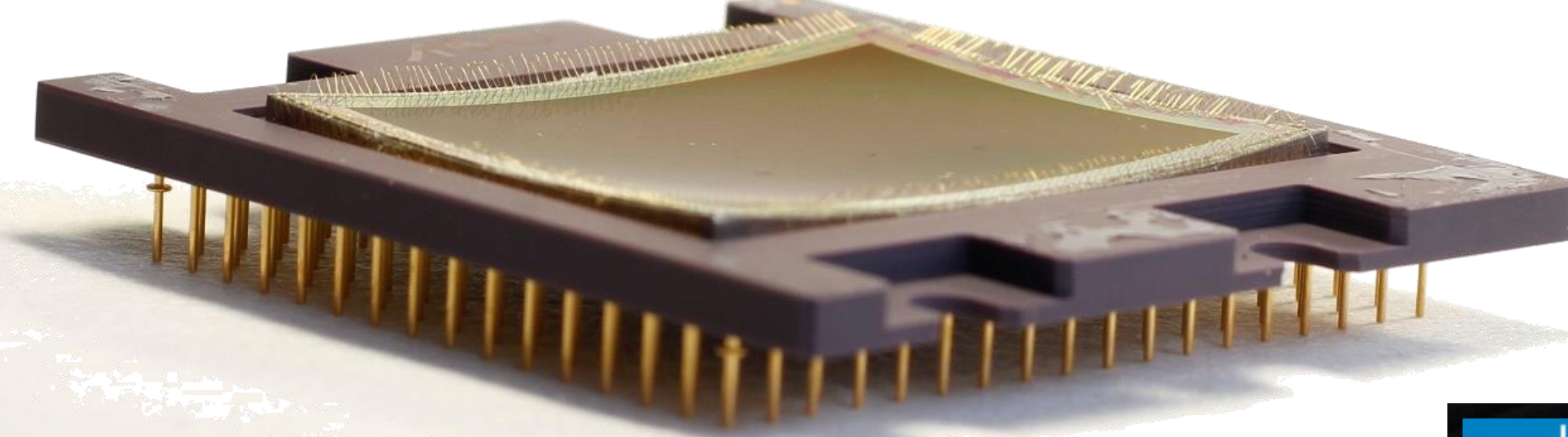

CMOS visible sensor

Radius of curvature CC $150 \mathrm{~mm}$

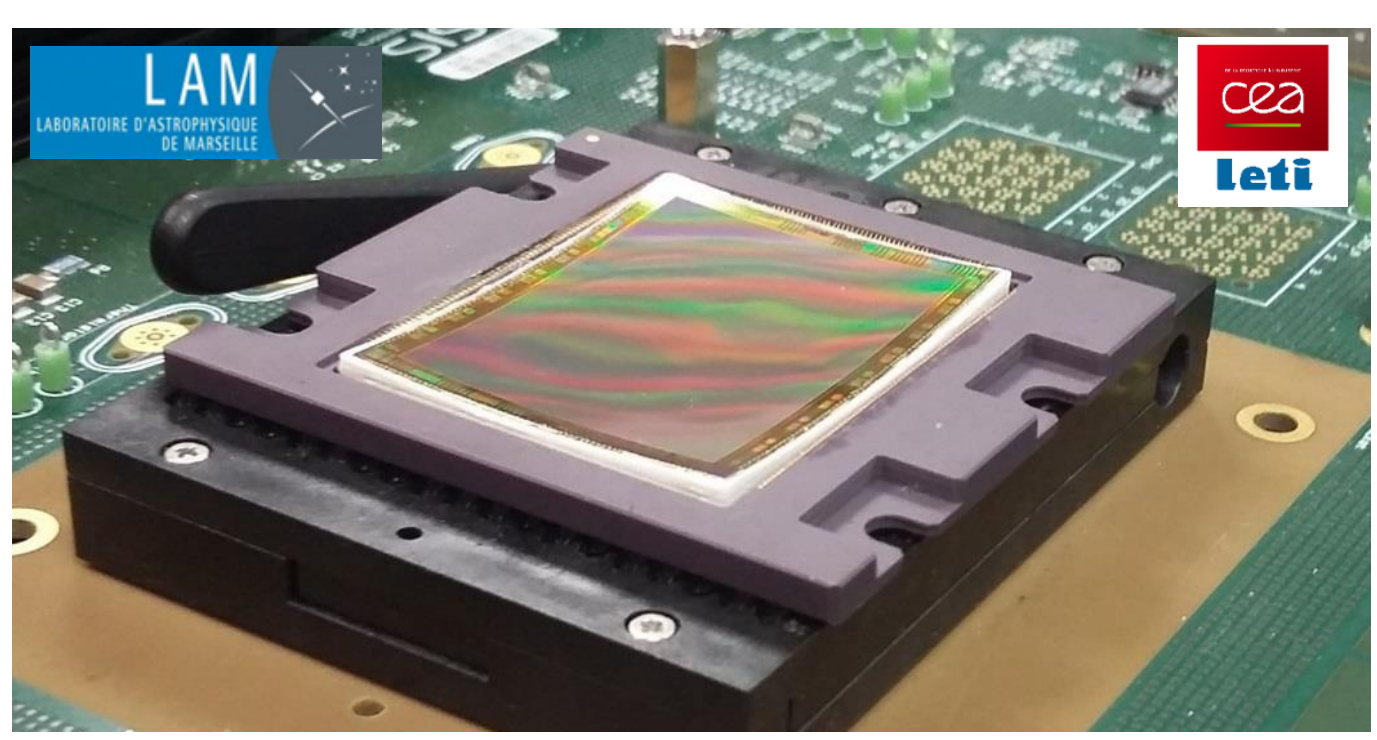


Full frame $24 \times 32 \mathrm{~mm}^{2}$

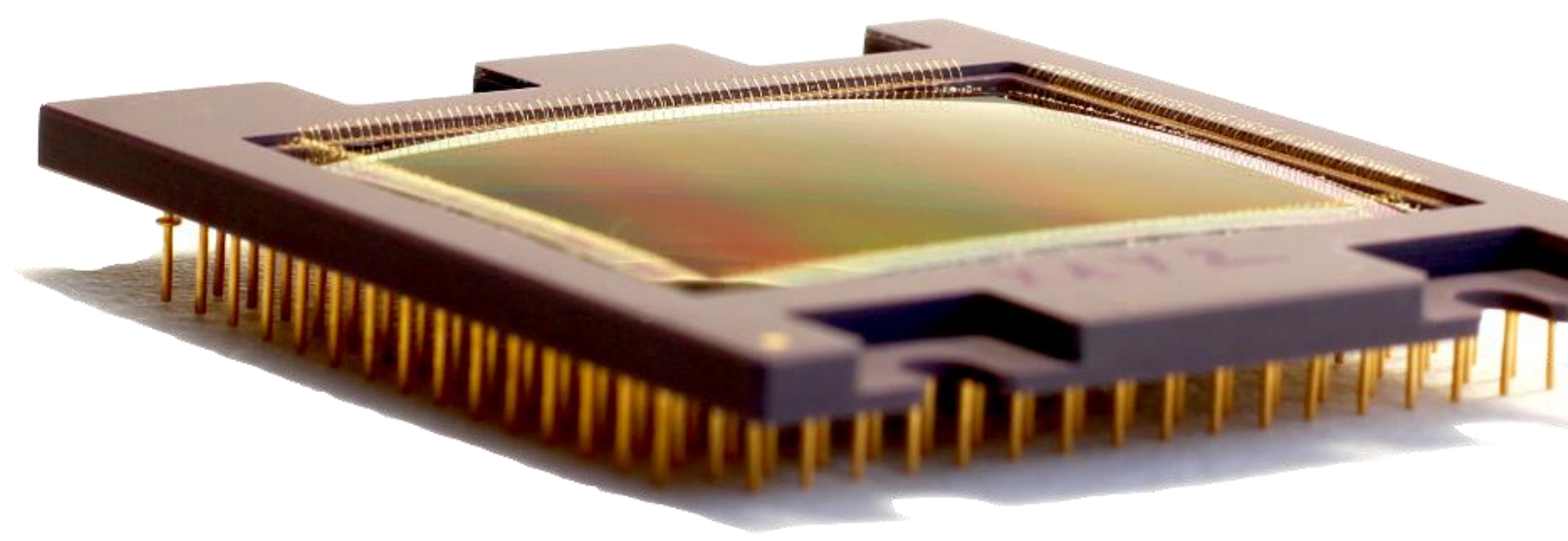

Radius of curvature CX $280 \mathrm{~mm}$

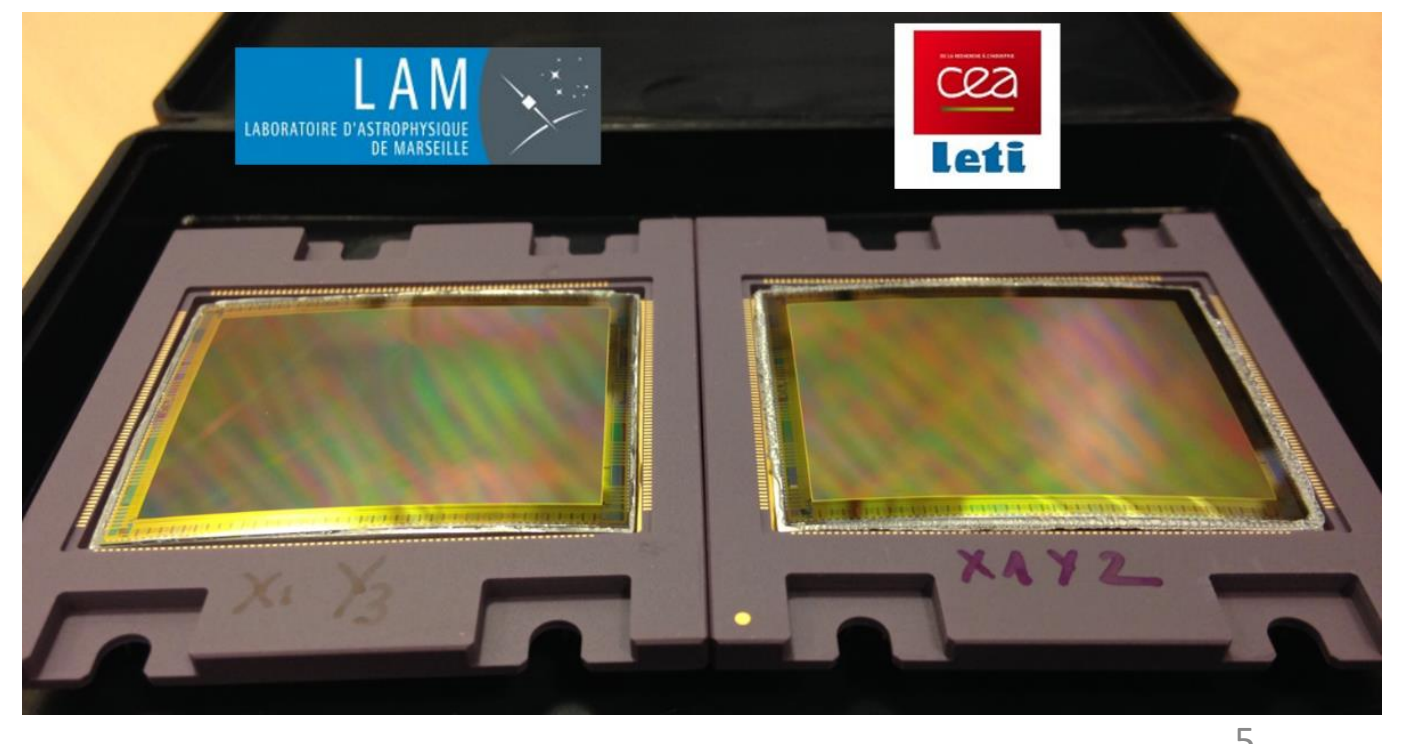




\section{CURVED SENSORS}

Comparison between flat and curved (ccv) samples

Performance characterization@LAM (300 K)
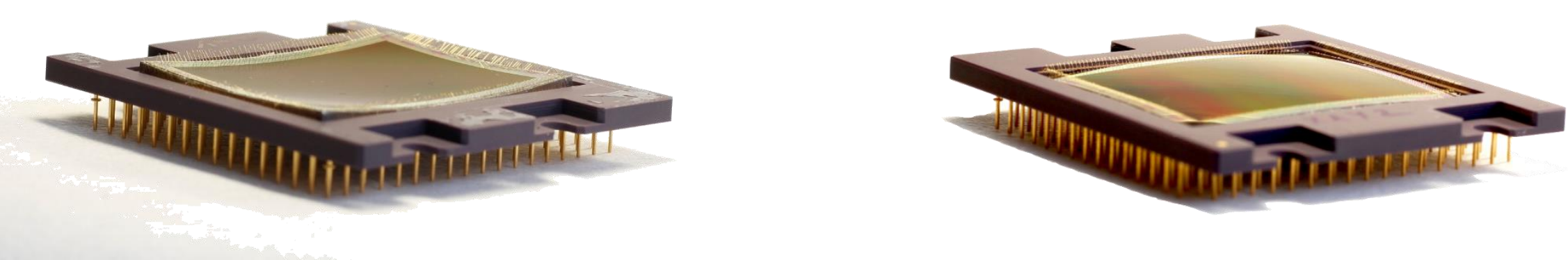
Curved CMOS performances: Dark and flat field signals
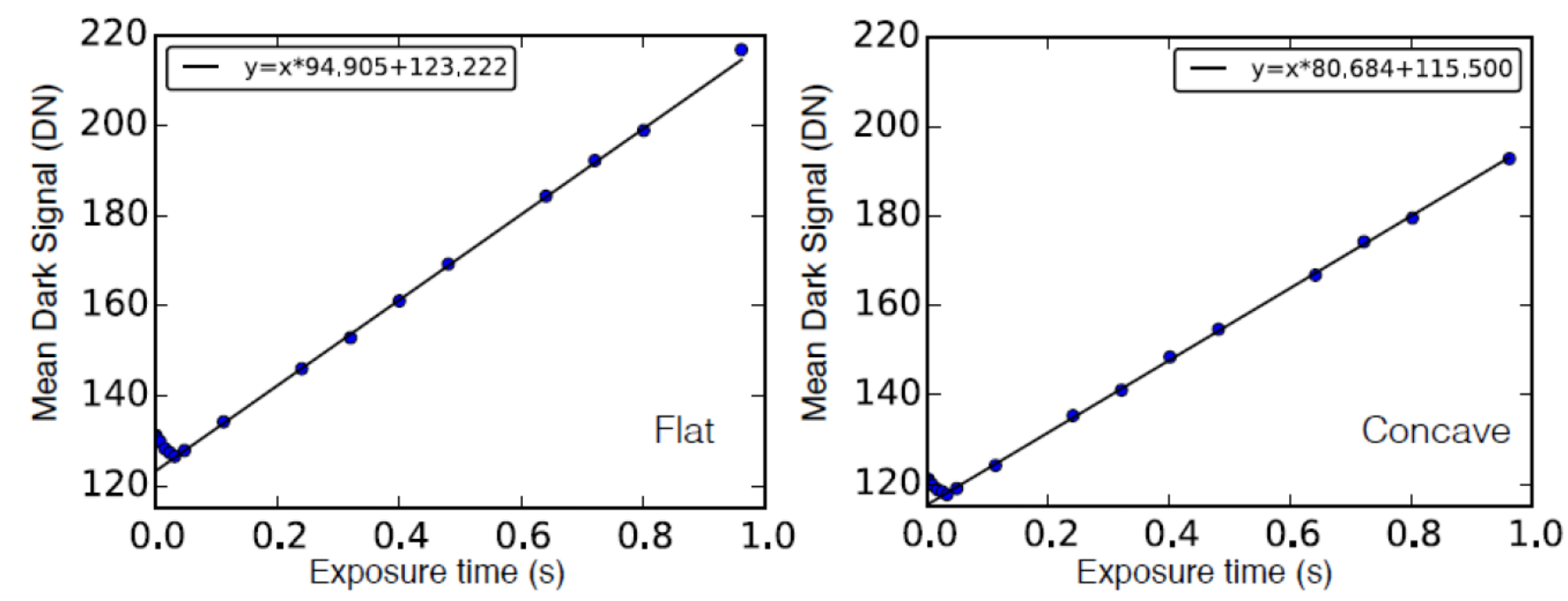

Mean dark signals / exposure time

$\rightarrow$ similar performance
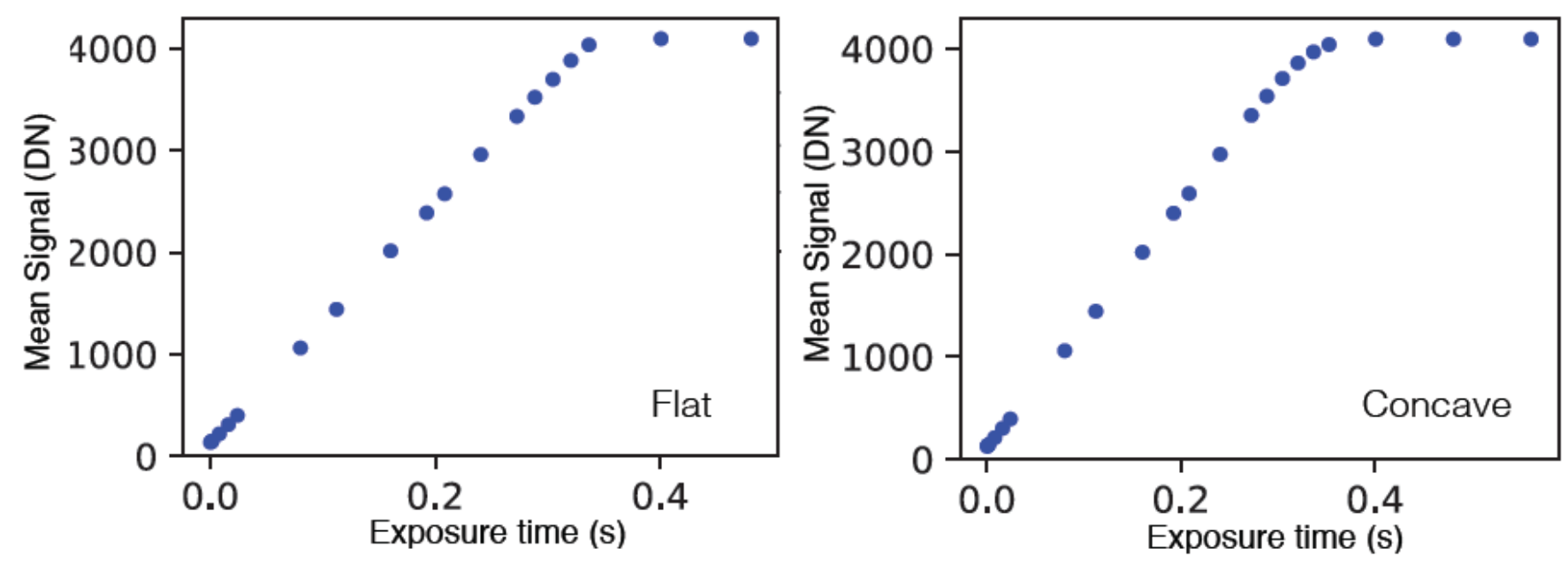

Mean flat field signals / exposure time $\rightarrow$ identical performance 
Curved CMOS performances: Temporal and Read out noises
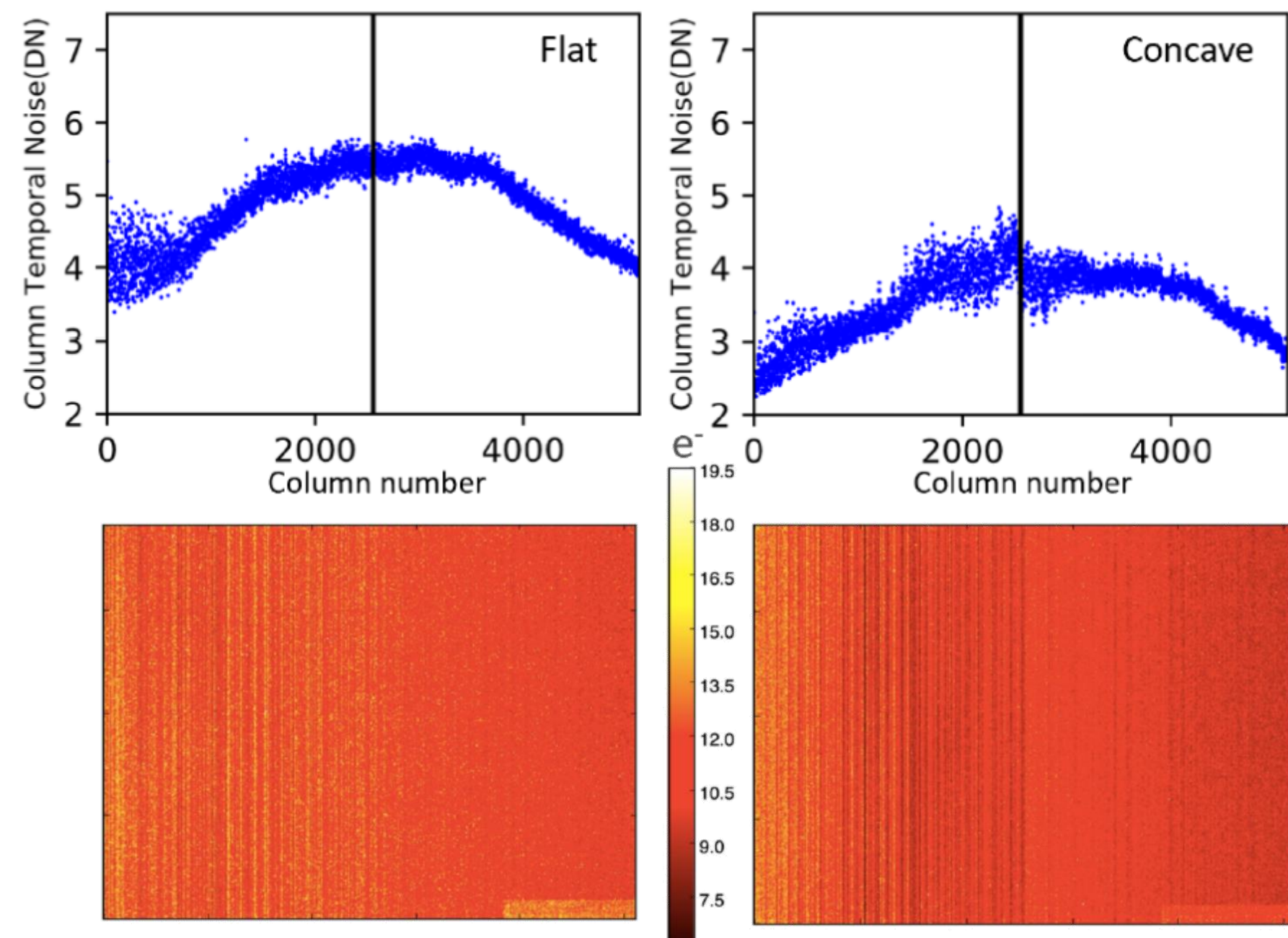

Column temporal noise

Median dark exposure at $0.0002 \mathrm{~s}$ $\rightarrow$ slightly better performance 


\section{$\mathrm{F}=\mathrm{CUS}$}

\section{CURVED SENSORS}

Comparison between flat and curved (ccv) samples

Curved CMOS have exactly same performance than flat sensors

Slightly better in term of noises (to be confirmed)
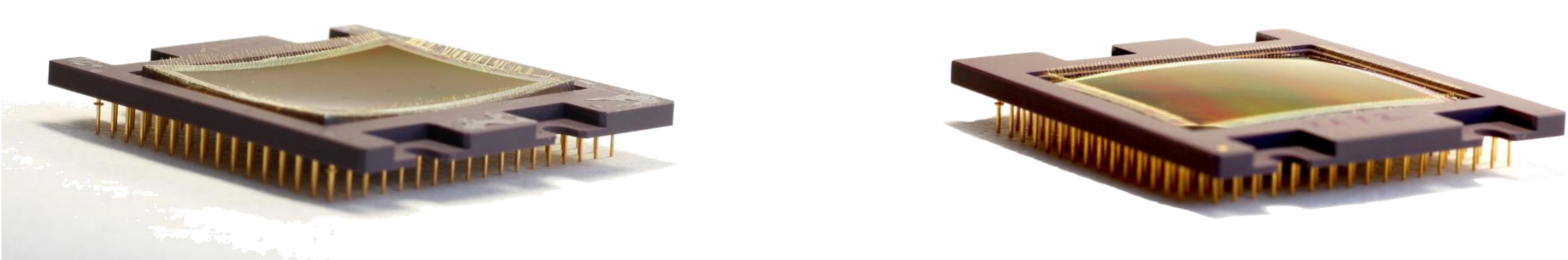
FISH-EYE DEMONSTRATOR

180 degrees field of view, adapted to concave or convex sensors

\section{DEGREES E2V-BASED MINI-IMAGER}

Prototype demonstrating utmost performance with E2V curved sensor 


\section{Fish-eye demonstrator}

Comparison based on CANON design

$>$ Two optimized optical designs

- one lab prototype

- one pre-industrial prototype

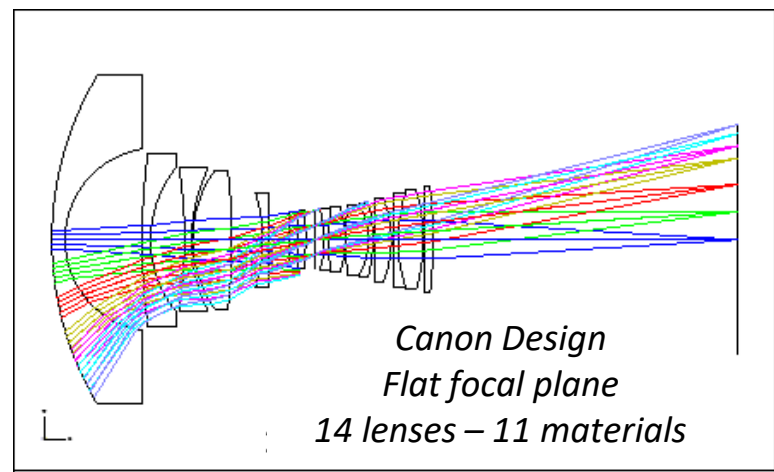

Increased optical quality (spot reduction up to 50\%) Save about $30 \%$ of optical surfaces
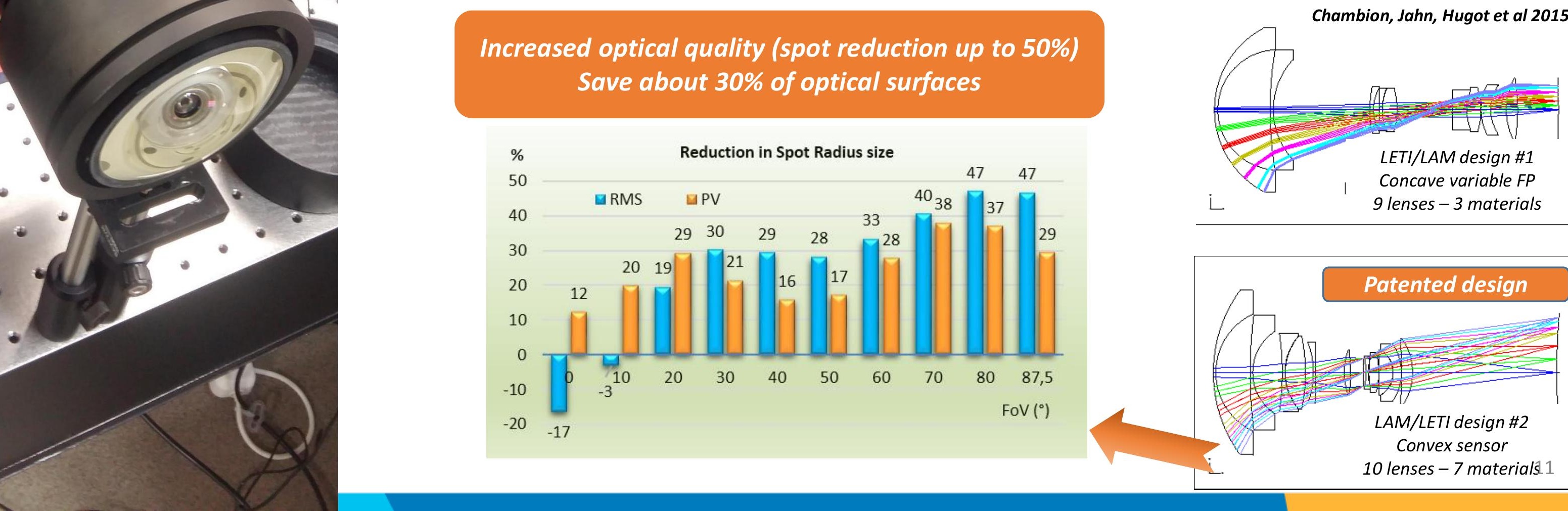

Concave variable FP

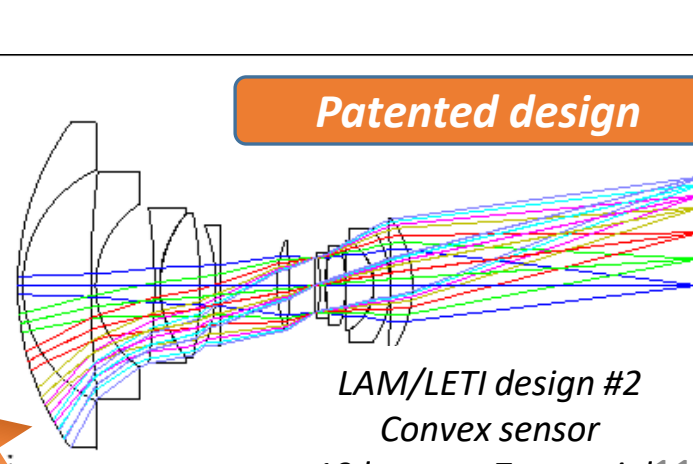


Fish-eye demonstrator - performance
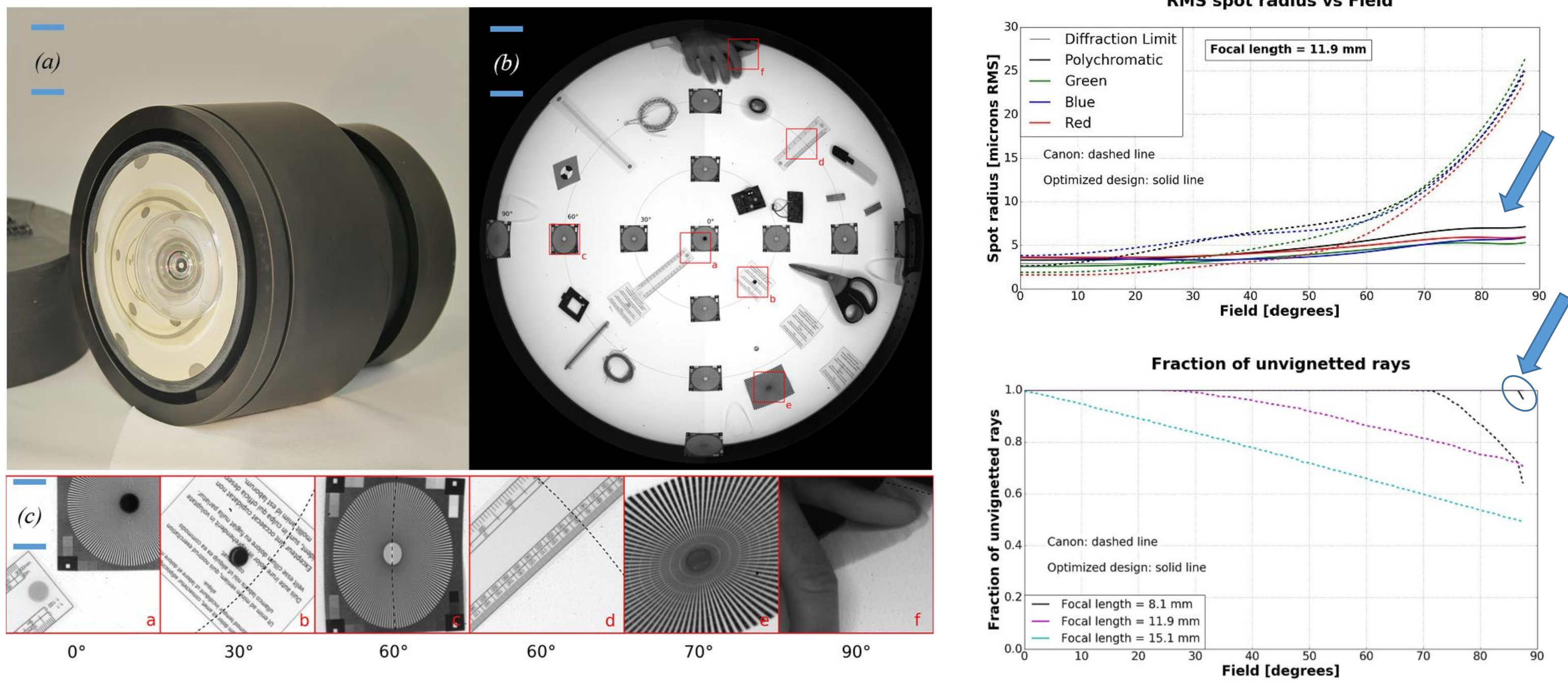


\section{Wide-field small objective}

Curved Teledyne E2V EV76C560 $1 / 1.8$ " sensor

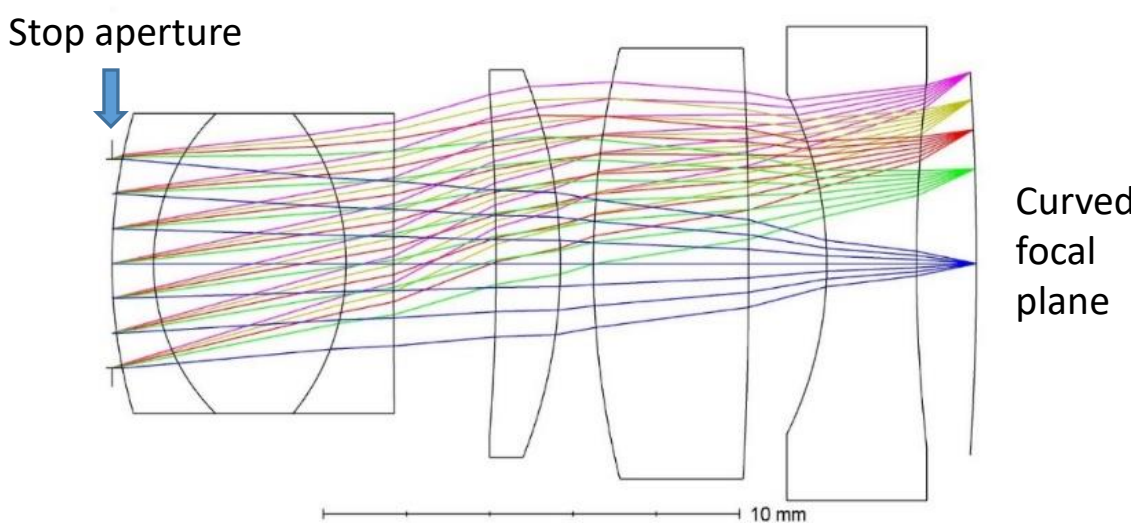

40 degrees FoV - 20mm long

MTF Design with curved sensor. WD $=2 \mathrm{~m}$

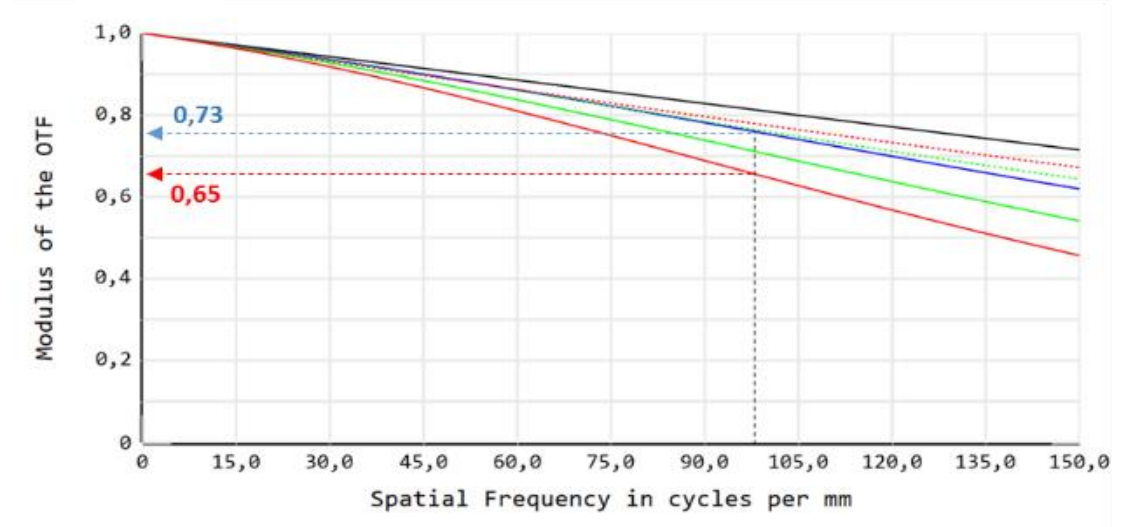

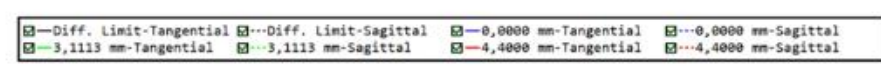

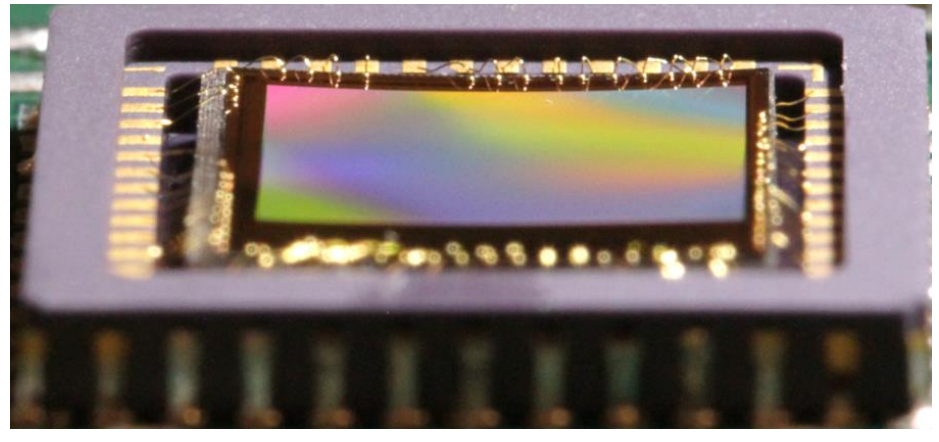

slightly better Gain, Read out noise and Dark current.

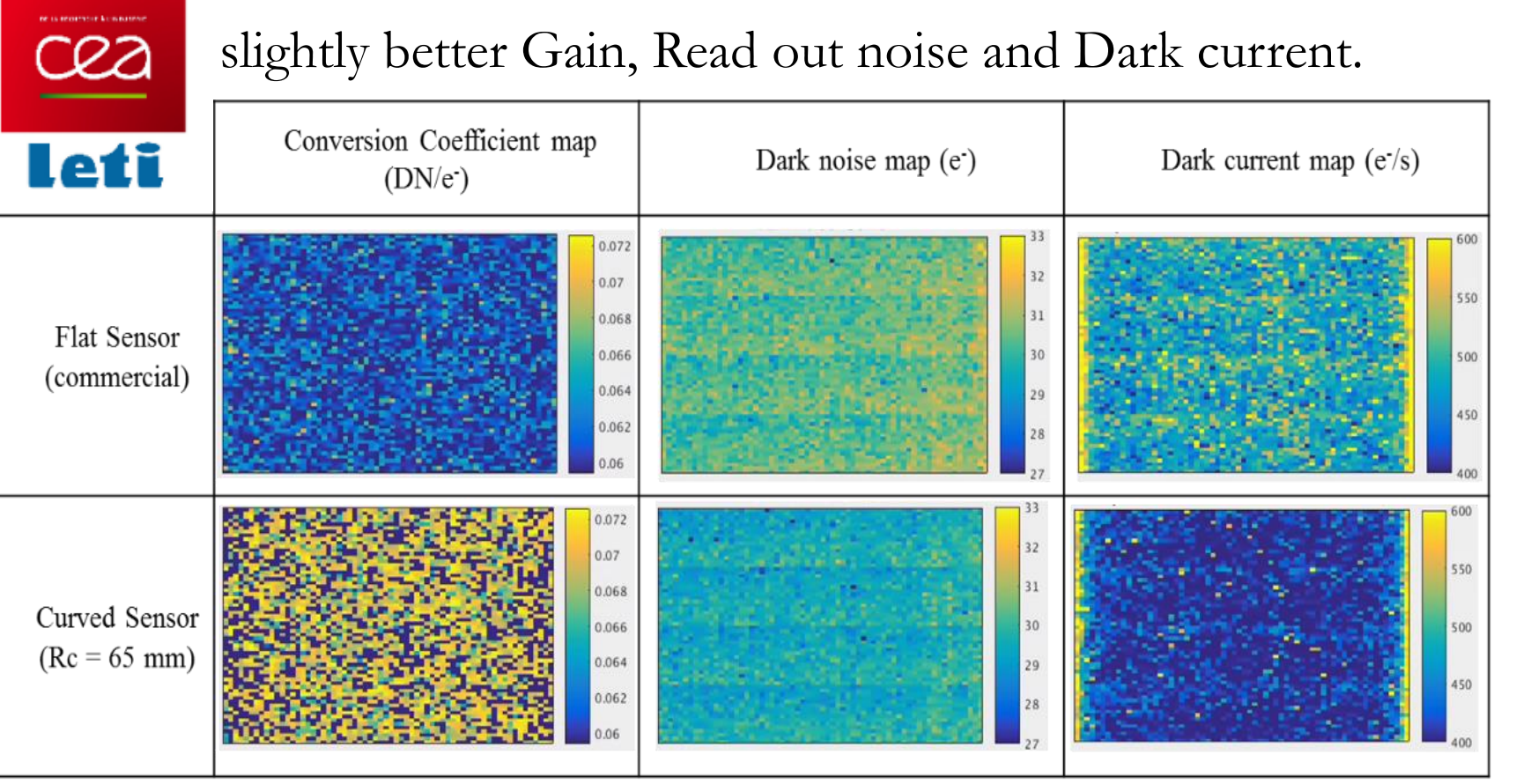




\section{Wide-field small objective}

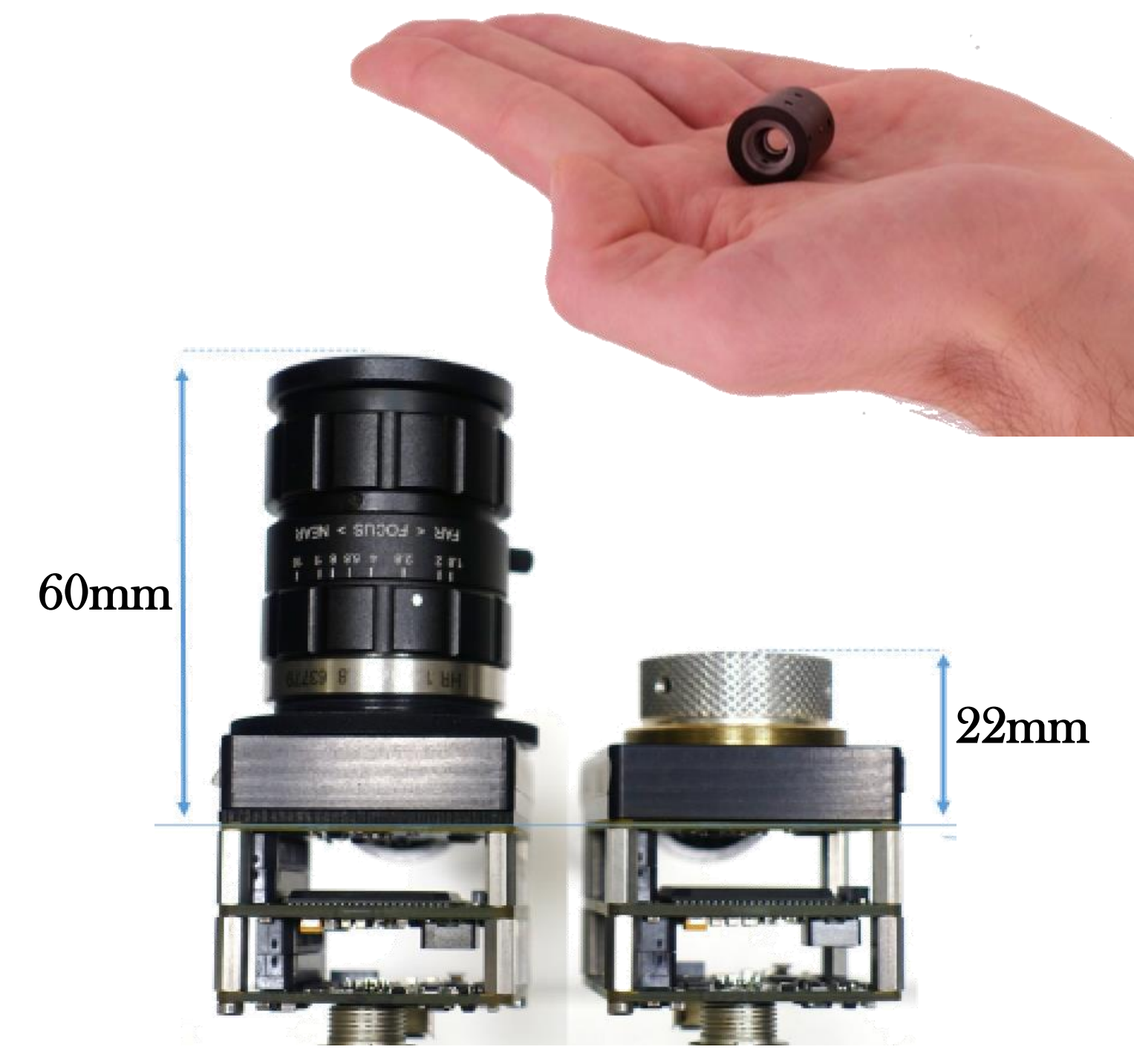

Comparison with commercial system (TECHSPEC ${ }^{\circledR}$ High resolution lens /63779)

\begin{tabular}{|c|c|c|c|c|c|}
\hline $\begin{array}{c}\text { Contrast } \\
50 \% / 0^{\circ} / \\
\mathrm{f} / 2,8\end{array}$ & Distorsion & FoV & Lenses & $\begin{array}{c}\text { Total } \\
\text { length }\end{array}$ & Curvature \\
\hline $\begin{array}{c}180 \mathrm{cycles} \\
\mathrm{mm}\end{array}$ & $<3 \%$ & $42^{\circ}$ & 10 & $60 \mathrm{~mm}$ & +inf. (flat) \\
\hline
\end{tabular}

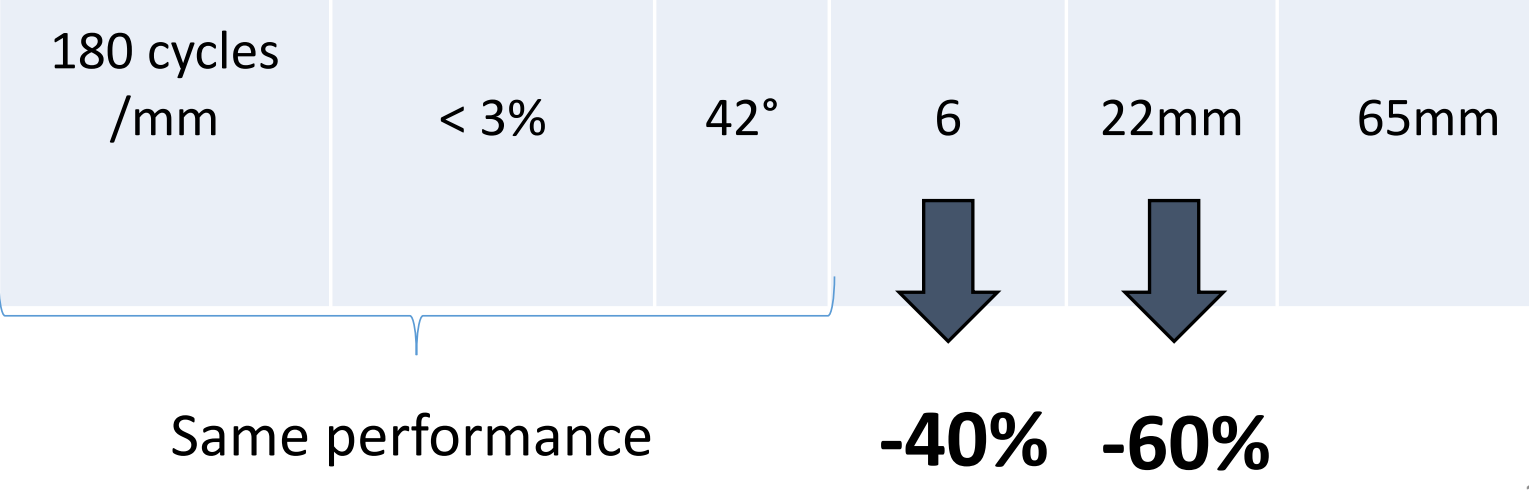


Component: TRL4, Patent Gaeremynck et al 2015 (Post doc FOCUS)

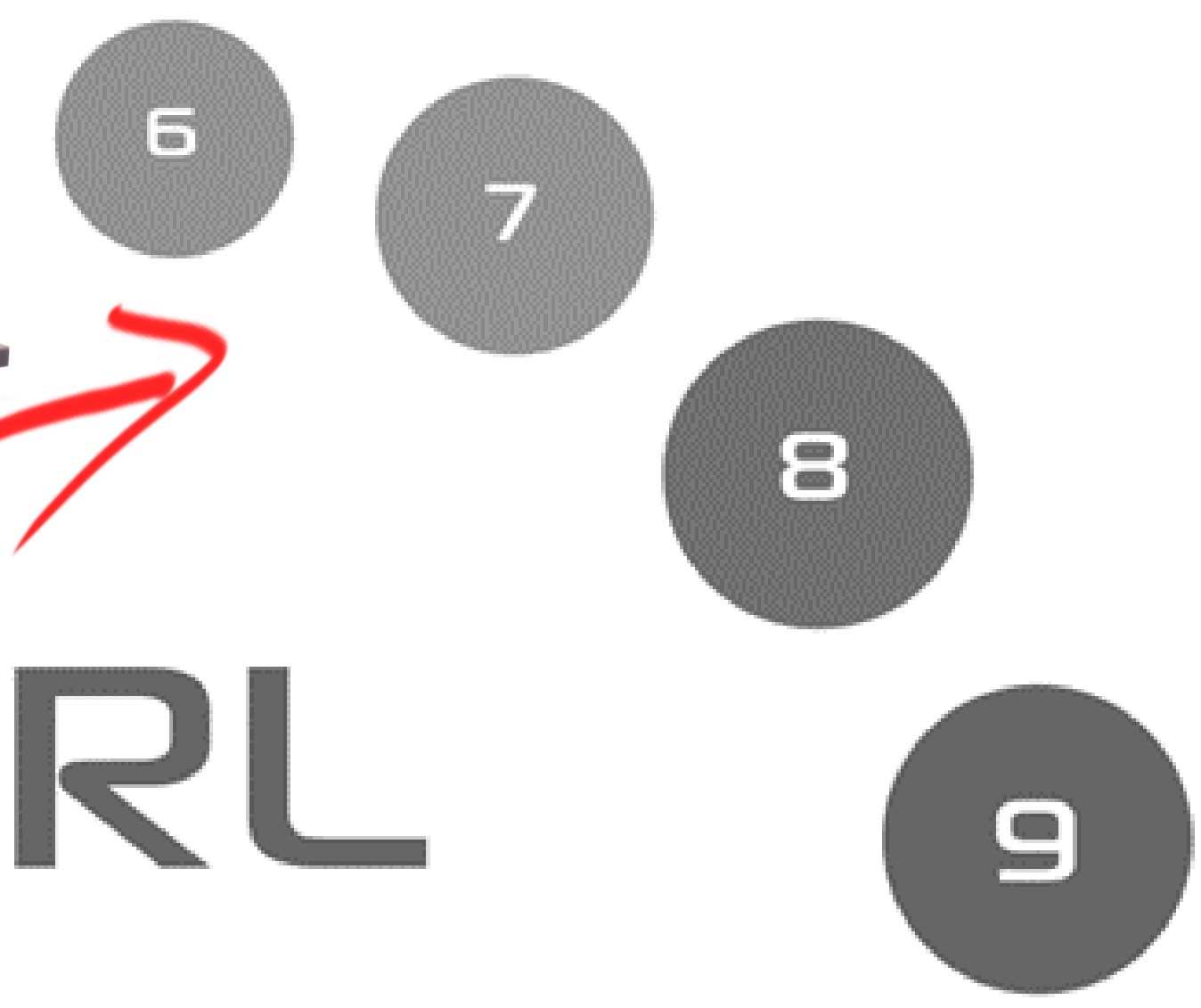




\section{(Aix Marseille}

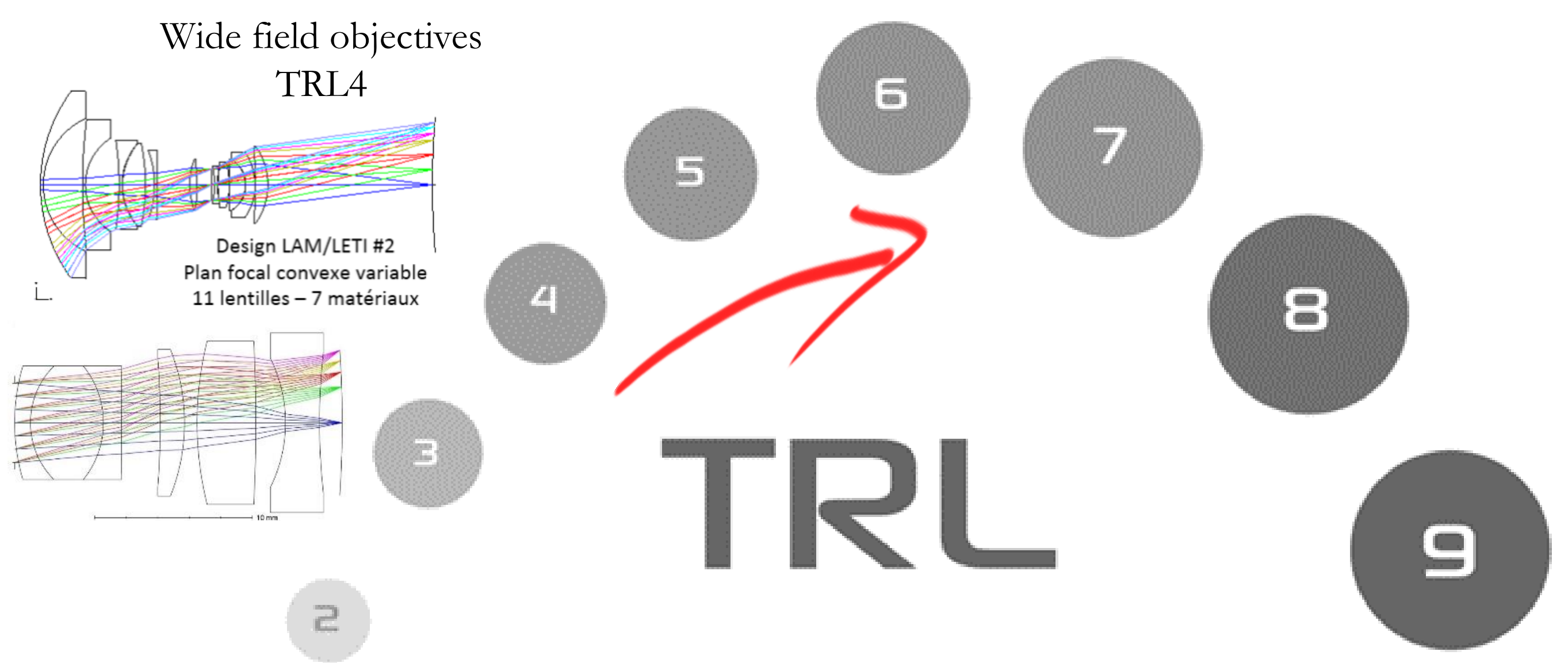




\section{SUMMARY}

\section{Exquisite performance}

$\rightarrow$ Components level: similar performance wrt flat sensors

$\rightarrow$ System level: Compact / high performance optical systems enabled

TRL4 for the components and systems

$\rightarrow$ Ready to start vacuum/thermal qualifications

$\rightarrow$ ESA GSTP already identified

Large format CCDs to be developed in 2019

$\rightarrow$ Funded by the European Astronomical Society

$\rightarrow$ Discussions with Teledyne E2V

$\rightarrow$ Concept of Blue-MUSE for ESO VLT

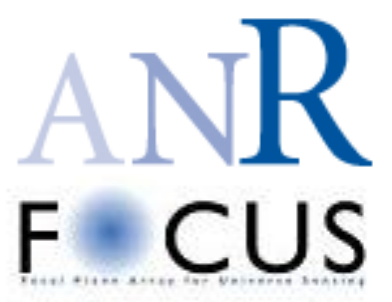




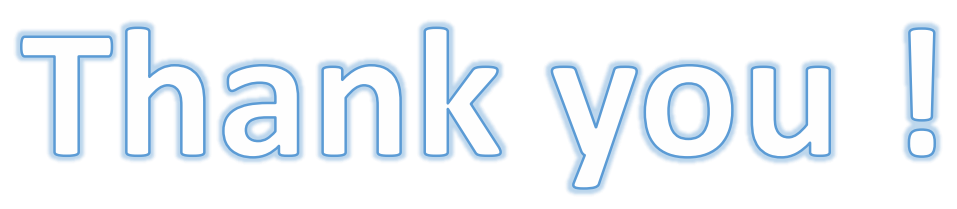
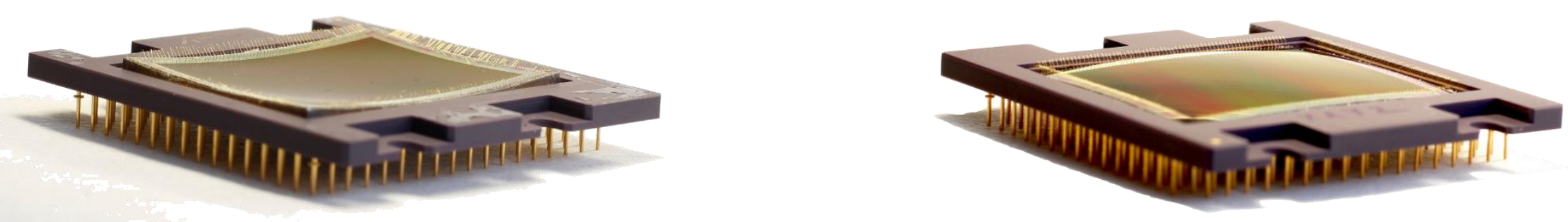

$\mathrm{F}=\mathrm{CUS}$ 